Document downloaded from:

http://hdl.handle.net/10251/80319

This paper must be cited as:

Vikingsson, L.; Vinals Guitart, A.; Valera Martínez, A.; Riera Guasp, J.; Vidaurre, A.; Gallego Ferrer, G.; Gómez Ribelles, JL. (2016). Local deformation in a hydrogel induced by an external magnetic field. Journal of Materials Science. 51(22):9979-9990. doi:10.1007/s10853-016-0226-8.

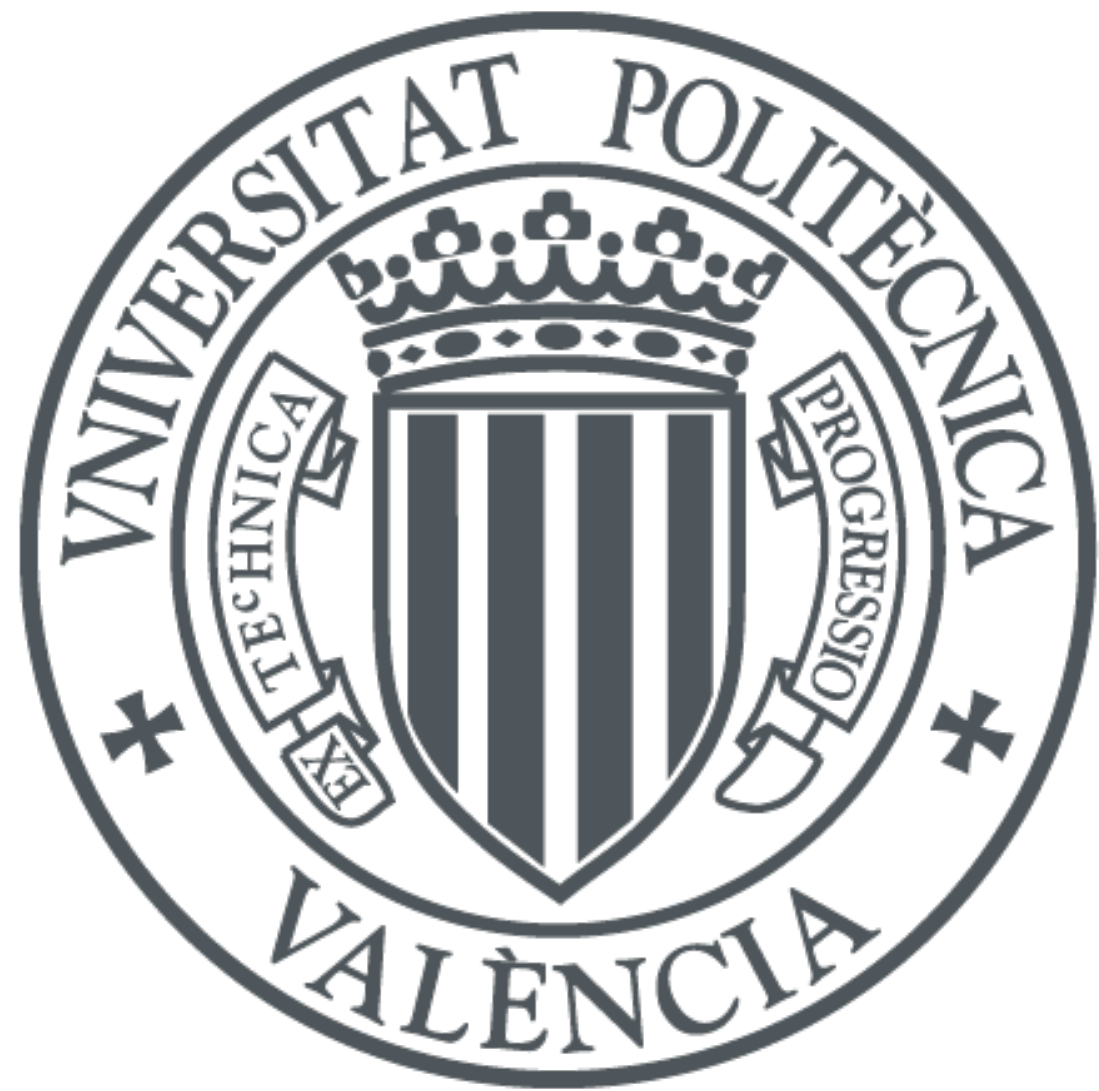

The final publication is available at

http://dx.doi.org/10.1007/s10853-016-0226-8

Copyright Springer Verlag (Germany)

Additional Information 


\title{
Local deformation in a hydrogel induced by an external magnetic field.
}

\author{
L. Vikingsson ${ }^{\dagger, 1, \star}$, A. Vinals-Guitart ${ }^{\dagger, 1}$, A. Valera-Martínez ${ }^{1}$, J. Riera ${ }^{2}$, A. \\ Vidaurre $^{1,3}$, G. Gallego Ferrer ${ }^{1,3}$, J.L. Gómez Ribelles ${ }^{1,3}$
}

\section{${ }^{\dagger}$ Equal contribution}

${ }^{1}$ Center for Biomaterials and Tissue Engineering (CBIT), Universitat Politècnica de València, 46022 Valencia, Spain.

2 Instituto Universitario de Matemática Multidisciplinar, Universitat Politècnica de València, 46022 Valencia, Spain.

${ }^{3}$ Networking Research Center on Bioengineering, Biomaterials and Nanomedicine (CIBER-BBN), Valencia, Spain

*Corresponding author. Tel.: +46763152128; Fax: +34 963877276. E-mail: linevikingsson@gmail.com

\section{Abstract}

The aim of this study is to prove the feasibility of a system able to apply local mechanical loading on cells for tissue engineering applications. This experimental study is based on a previously developed artificial cartilage model with different concentrations of Poly(Vinyl Alcohol), PVA, that simulates the cartilage extracellular matrix, ECM. Poly(L-lactic Acid), PLLA, microspheres with dispersed magnetic nanoparticles, MNP, were produced with an emulsion method. These microspheres were embedded in aqueous PVA solutions with varying concentration to resemble increased viscosity of growing tissue during regeneration. The ability to induce a local deformation in the ECM was assessed by applying a steady or an oscillatory magnetic field gradient to different PVA solutions. PLLA microparticle motion was recorded, and the images were analyzed. Besides, PVA gels and PLLA microparticles were introduced into the pores of a Polycaprolactone scaffold, and the 
microparticle distribution and the mechanical properties of the construct were evaluated. The results of this experimental model show that the dispersion of PLLA microparticles containing MNPs, together with cells in a supporting gel will allow applying local mechanical stimuli to cells during tissue regeneration. This local stimulation can have a positive effect on the differentiation of seeded cells and improve tissue regeneration.

Key words: Magnetic nanoparticles, Microparticles, Polycaprolactone, Poly(lactic acid), Poly (Vinyl Alcohol), Local stimulation of cells.

\section{Introduction}

Articular cartilage is a tissue with relatively few cells embedded in a dense extra cellular matrix mainly composed of collagen type II and proteoglycans. ${ }^{7,29}$ The interactions between cells and matrix are controlled by signal factors and a complex flow and force controlled system. The physical stimuli and the cell response is a multiform feedback system that has been studied at a molecular, cellular and higher tissue level. ${ }^{9,28,2,1}$ A major component in the ECM of articular cartilage are the negatively charged glucosoaminoglycans. These fixed charge densities give the tissue electromechanical properties. The flow of free electrolytes through the medium containing fixed charges creates physicochemical responses and charge-dependent osmotic swelling pressure. Upon external mechanical loading, electric potentials are developed with the chondrocytes being exposed to these electrochemical events. This signal transduction mechanism is directly dependent on the external mechanical loading of the joint. ${ }^{23,24}$ The chondrocytes respond to the mechanical stimuli by multiple regulatory pathways, with consequences on transcription, translation and post-translational modifications that further control extra cellular matrix production and function. ${ }^{4,34}$ Integrins, growth factor receptors, cytoskeletal filaments, nuclei, stretch-activated ion channels and cartilage ECM proteins are just some of the signaling molecules that contribute to biosynthesis, remodeling, or repair of the tissue as mechanotransduction response to mechanical loading. ${ }^{14,15}$

Tissue Engineering is an integrative area of biotechnology and engineering that 
focuses on restoring or improving tissue function with cells and materials. ${ }^{13}$ Today's tissue engineering strategies apply natural or synthetic materials with or without a previously seeded cell source to regenerate damaged tissue. A major challenge in cartilage engineering is to design structures that transmit adequate mechanical signals to cells in the scaffolds, as well as diminishing the difference in compression forces from surrounding tissue. ${ }^{13}$

Beyond the physiological compression subjected to the joint, the ability for external control of specific cellular components in vivo is a highly interesting research area that can lead to tools for manipulating cell growth and tissue differentiation. Magnetic actuation on cellular behavior has been investigated, ${ }^{5}$ and magnetic micro- and nanoparticles have gained interest for their ability for remote control of cellular behavior, ${ }^{32,33}$ e.g. magnetic tweezers attached to integrin receptors on a cell surface. ${ }^{38}$ By applying a rotating magnetic field, the particles can twist and hence the force required to twist the particles is related to the mechanical properties of the cytoskeleton. Magnetic tweezers have also been used to activate mechanosensitive ion channels to study and control the production of specific proteins. ${ }^{27,3}$ The cellular effect of osteoblasts and fibroblasts in magnetic polymer nanofibers has been studied, and it was observed that the cells that aligned to the applied magnetic field showed increased cell proliferation. ${ }^{21}$

In this study we have embedded magnetic nanoparticles in Poly(L-lactic acid), PLLA, microparticles produced via the emulsion method. The microparticles loaded with nanoparticles have been introduced in different Poly(vinyl alcohol), PVA, aqueous solutions. In previous studies, we have shown that it is possible to tailor the mechanical properties of Polycaprolactone, PCL, scaffolds filled with PVA, by applying cycles of freezing and thawing, and reach mechanical modulus of articular cartilage ${ }^{35,36,37}$ Several authors refer to PVA gels as cartilage scaffolds or tissue substitutes. ${ }^{20,10}$ This study aims at experimentally studying the movement of the PLLA microparticles embedded in different medium densities that resemble the growing ECM. Then an external magnetic field has been applied to the nanoparticles in water and aqueous solutions with varying PVA concentrations. Furthermore, the nanoparticle loaded PLLA microspheres in PVA hydrogel have been injected into porous PCL, scaffolds. We further explore our previously developed artificial cartilage 
model, applying it on scaffolds loaded with PLLA microspheres with embedded MNP. The physicochemical properties such as the mechanical properties and morphology of the PCL/PVA with PLLA/MNP spheres have been characterized.

\section{Materials and Methods}

\subsection{Materials}

Polycaprolactone, average molecular weight $80 \mathrm{kDa}(\mathrm{Mw} / \mathrm{Mn}<2)$, was obtained from Sigma Aldrich (Spain). Microspheres of Elvacite 2043, a mixture of low molecular weight Poly(ethyl methacrylate), and Poly (methyl methacrylate), PEMA/PMMA, with diameters ranging from 120 to $200 \mu \mathrm{m}$ were purchased from Lucite International (USA). 1.4 Dioxane (Sigma Aldrich, Spain) was used as solvent for PCL, and ethanol, EtOH, (99\% pure Scharlab, Spain) was used to dissolve the dioxane and PEMA/PMMA porogen particles in the freeze extraction and particle leaching process. Poly(Vinyl alcohol), average Mw $130 \mathrm{kDa}, 99+\%$ hydrolyzed was purchased from Sigma Aldrich (Spain) and used in hydrogel preparation and as aqueous phase in the oil/water emulsion method for microparticle synthesis. Poly(L-lactic acid), PLLA, (Purasorb PL-18 medical grade, PURAC) with average molecular weight 217$250 \mathrm{kDa}$ was used for microparticle fabrication. Chloroform, ACS reagent grade, stabilized with ethanol (99.8\%), and tetrahydrofuran, THF, ACS reagent grade, 99.8\% were purchased from Sigma Aldrich (Spain) and used as solvent for PLLA. THF, GPC grade from Sharlau, was used as eluent for molecular weight determination. Superparamagnetic hydrophobic magnetic nanoparticles, MNP, (EMG 1300) were purchased from Ferrotec Ferrofluid as dry powder. All the chemicals were used as received and with no further modification.

\subsection{Microparticle synthesis}

MNPs were dispersed in a $2 \% \mathrm{w} / \mathrm{v}$ PLLA solution in chloroform with a concentration of $5 \% \mathrm{w} / \mathrm{w}$ MNP to PLLA (hereafter called PLLA/MNP dispersion). All the procedure was conducted under a methacrylate hermetic box to assure no MNP escape by air. PLLA/MNP microparticles were fabricated via an oil/water emulsion method. The oleic phase consisted of $2 \% \mathrm{w} / \mathrm{v}$ PLLA/MNP dispersion in chloroform and the aqueous phase of $4 \% \mathrm{w} / \mathrm{v}$ Poly(Vinyl alcohol) solution. Briefly: $20 \mathrm{~mL}$ of PLLA/MNP 
dispersion was added drop wise via a syringe pump into $200 \mathrm{~mL}$ of PVA solution at constant stirring at $750 \mathrm{rpm}$ with $1 \mathrm{~mL} / \mathrm{min}$ pump rate. $150 \mathrm{~mL}$ of deionized water was added to assist with solvent evaporation, and the mixture was stirred for 24 hours. After stirring, the resulting microparticle dispersion was washed twice with water, filtered with ethanol through a $50 \mu \mathrm{m}$ filter, air-dried and vacuum dried prior to microparticle collection.

In order to improve their hydrophilicity, the microparticles were subjected to plasma treatment inside a Piccolo (Plasma Electronic) Microwave plasma chamber. The plasma treatment parameters were: Argon gas, $50 \mathrm{~Pa}$ initial gas pressure, with a gas flow rate of $160 \mathrm{sccm}$ Ar, for 600 seconds. Treatment times from 60 to 600 seconds were previously assayed to assess the limit tolerable by the microparticles, and no alteration was seen at any point throughout this range. PLLA microparticles without MNP were prepared following the same protocol.

\subsection{Scaffold preparation}

$15 \% \mathrm{w} / \mathrm{w}$ and $20 \% \mathrm{w} / \mathrm{w}$ PCL solutions in 1.4 dioxane were prepared by continuous stirring for 24 hours until transparent and homogeneous solutions were obtained. Scaffolds with a double micro and macro porosity were fabricated according to previous studies. ${ }^{19,31,35,37}$ Briefly: PCL solution was mixed with PEMA/PMMA microspheres in a weight ratio of $1: 1.25$. The mixture was immediately frozen with liquid nitrogen. After approximately one minute, the frozen polymer was immersed in precooled $\mathrm{EtOH}$ and kept at $-20^{\circ} \mathrm{C}$ for three days with daily solvent changes. To remove the PEMA/PMMA spheres the scaffolds were washed in $\mathrm{EtOH}$ at $40^{\circ} \mathrm{C}$ for 8 days, changing the EtOH twice every day until no traces of PEMA/PMMA microspheres were seen. The scaffolds were cut with circular stamps and surgical scalpels $5 \mathrm{~mm}$ in diameter and $2 \mathrm{~mm}$ high.

\subsection{Scaffold/hydrogel constructs}

The scaffold/hydrogel constructs were obtained as described previously. ${ }^{35,36,37,26}$ Briefly: a $10 \% \mathrm{w} / \mathrm{v}$ aqueous solution of PVA was prepared by stirring at $80^{\circ} \mathrm{C}$ for 2 hours and left to cool to room temperature. The viscous solution was then poured 
into a 48 multi-well cell culture plate. Pure PVA hydrogels were obtained by freezing the solution at $-20^{\circ} \mathrm{C}$ for 12 hours and then thawed back to room temperature for 8 hours. Water immersion of the hydrophobic PCL scaffolds was carried out by changing the solvent from $\mathrm{EtOH}$ to water. To make sure that all the pores were filled with water, the scaffolds were put in water and subjected to continuous vacuum extraction. The plasma treated PLLA microparticles were dispersed in PVA solution at $50^{\circ} \mathrm{C}$ and homogenized by sonication. PVA solution filled with PLLA microparticles was then introduced into water immersed PCL scaffolds by vacuum injection and the scaffolds were subjected to a number of successive freezing and thawing cycles as described for pure PVA hydrogels. All samples were left in water 24 hours before mechanical testing.

The porosity of the scaffolds was obtained by dividing the volume of pores ( $\left.V_{\text {pores }}\right)$ by total sample volume $\left(V_{\text {total }}\right)$, with the sum of scaffold volume $\left(V_{\text {scaffold }}\right)$ and pore volume being in accordance with equation 1 . The volume of pores was calculated by scaffold mass filled with PVA ( $\left.m_{\text {withPVA }}\right)$, subtracting the dry scaffold mass $\left(m_{\text {dry }}\right)$, and divided by the PVA solution density ( $\left.\rho_{\text {PVAsolution }}\right)$, according to equation 2. PVA solution density was estimated from a 10\% PVA aqueous solution with a pure PVA density of $1.30 \mathrm{~g} / \mathrm{cm}^{3}{ }^{11}$ The scaffold volume was calculated by dividing the dry scaffold mass by $\mathrm{PCL}$ density $\left(\rho_{P C L}\right.$ ), equation $3 . \mathrm{PCL}$ density is $1.146 \mathrm{~g} / \mathrm{cm}^{3} .{ }^{17}$ Porosity was calculated for 6 replicates of PCL scaffold filled with PVA solution and caution was taken to eliminate any excess PVA on the scaffold surface.

$$
\begin{gathered}
\text { Porosity }=\frac{V_{\text {pores }}}{V_{\text {total }}}=\frac{V_{\text {pores }}}{V_{\text {scaffold }}+V_{\text {pores }}} \\
V_{\text {pores }}=\frac{m_{\text {with } P \text { VA }}-m_{d r y}}{\rho_{P V A s o l u t i o n}} \\
V_{\text {scaffold }}=\frac{m_{d r y}}{\rho_{P C L}}
\end{gathered}
$$

\subsection{Differential Scanning Calorimetry}

DSC heating scans (PYRIS-DSC 8000, Perkin Elmer) were conducted to measure PLLA microparticle crystallinity and the effect of nanoparticle dispersion and plasma treatment of the microparticles. The scans were run between $0^{\circ} \mathrm{C}$ to $200^{\circ} \mathrm{C}$ at a 
heating rate of $20^{\circ} \mathrm{C} / \mathrm{min}$, and from $200^{\circ} \mathrm{C}$ to $0^{\circ} \mathrm{C}$ at a cooling rate of $40^{\circ} \mathrm{C} / \mathrm{min}$. The specific melting enthalpy, $\Delta h_{m}$, value used for fully crystalline PLLA was $93.7 \mathrm{~J} / \mathrm{g} .{ }^{22}$

\subsection{Scanning and Field Emission Electron Microscopy}

Morphology of water immersed PCL scaffolds filled with PVA hydrogel and PLLA/MNP microparticles, and the pure PVA hydrogel loaded with PLLA/MNP microparticles, subjected to 6 cycles of freezing and thawing, was observed in a cryogenic scanning electron microscope (cryo-SEM) JSM-6300 (JEOL Ltd., Tokyo, Japan). Water immersed samples were rapidly frozen in liquid nitrogen, and fractured in the cryo-transfer system vacuum chamber. After water sublimation at $-90^{\circ} \mathrm{C}$, the sample was coated with gold in the same chamber, directly moved into the SEM column and examined.

Dry PLLA/MPN microparticles were observed in a Field Emission Scanning Electron Microscope, FESEM, device (Zeiss Auriga Compact). Images were taken at 2-5 kV with platinum coating for image contrast enhancement. For observation of nanoparticle dispersion inside the microparticles a PLLA/MPN microparticle was cross-sectioned by using a Focused Ion Beam, FIB, device. The FIB operates with a focused beam of Gallium ions at low beam currents for imaging and high beam currents for site specific sputtering, which allows precision milling of the sample to nanoscale. After sectioning, the particle was covered with carbon and observed in the FESEM attached to the FIB equipment.

\subsection{Mechanical testing}

The PCL scaffolds filled with PVA with and without PLLA microparticles were subjected to compression in a Microtest Universal Machine SCM 300095 with a custom made device that allows conducting the experiment in water-immersed conditions. The experiment was performed to $15 \%$ of strain with $1 \mathrm{~mm} / \mathrm{min}$ compression rate to simulate physiological conditions. ${ }^{6}$ The elastic modulus was calculated from the linear zone of the obtained stress-strain curve.

\subsection{Gel Permeation Chromatography (GPC)}

In order to ascertain the effect of the plasma treatment on the molecular weight of the polymer chains, a Gel Permeation Chromatography assay was conducted. Samples 
of microparticles with and without exposure to argon plasma were dissolved in THF at a concentration of $2 \mathrm{mg} / \mathrm{mL}$, and injected in a Waters HPLC/GPC device, composed of: Waters 1525 Binary Pump, Waters 2707 Autosampler, four serial Styragel HR columns, dimensions 7.8 x $300 \mathrm{~mm}$, and Waters 2414 Refractive Index Measurer. For each sample, two $20 \mu \mathrm{L}$ injection volume were run for excluding any disturbance during the measurement. THF was used as eluent at a flow rate of 1 $\mathrm{mL} / \mathrm{min}$ at $35{ }^{\circ} \mathrm{C}$. Polystyrene standards from Shodex (Showa Denko K.K., Kawasaki, Japan) were used to obtain a primary calibration curve. The values of the Mark-Houwink constants for PS $\left(k_{1}=0.000114 \mathrm{dL} / \mathrm{g}, \alpha_{1}=0.716\right)$ and PLLA $\left(k_{2}=\right.$ $\left.0.000545 \mathrm{dL} / \mathrm{g}, \alpha_{2}=0.73\right)^{8}$ were utilized to determine the molecular weight of the samples, $\mathrm{M}_{2}$, by the Mark-Houwink equation (equation 4).

$$
k_{1} M_{1}^{1+\alpha_{1}}=k_{2} M_{2}^{1+\alpha_{2}}
$$

\subsection{Magnetic Drag}

To imitate an in vivo situation and local mechanical stimulation of cells, a magnetic field was applied to different PVA solutions, representing growing ECM inside the scaffolds' pores. To imitate ECM production from cell ingrowth to cell differentiation, different concentrations of the PVA solution were tested. Water represents the initial state of cell invasion, and the lower concentrations of PVA act for ECM production and cell differentiation. PVA solution with PLLA/MNPs was placed in a $30 \mathrm{~mm}$ gap between two electromagnets. The electromagnets had nuclei composed of iron (relative permeability 860 ), $55 \mathrm{~mm}$ length and cross sectional area $18 \times 18 \mathrm{~mm}^{2}$, wrapped 500 times with copper wire. A circuit was designed to feed both electromagnets with two sinusoidal signals of $1 \mathrm{~Hz}$ and phase lag $\pi$ rad. The current intensity oscillated between 0 and $2.5 \mathrm{~A}$. The power supply was a NochstromNetzgerät 52155 with power $240 \mathrm{~W}$ and maximum intensity of $20 \mathrm{~A}$. Control drivers were fed with a symmetrical FAC-3638. Experimental setup was mounted onto the stage of a Nikon Eclipse TE100 microscope, by using a 10x Nikon lens with a final optical gain of 100x. The microscope was connected to a digital video camera (Mikrotron EoSens_CL_MC1362) that records images with a matrix size of $1280 \times 1024$ pixels, and a rate of 100 frames per second. The registered images were sent to a computer running MS Windows 7 , through a $\times 64 \times$ Celera-CLFull board, inserted in a PCl Expressx4 slot of the computer. Movement of PLLA/MNPs was 
followed by means of algorithms developed by our group based on image threshold techniques. All image processing was carried out using MATLAB R2010a (the MathWorks, Inc., Natick, MA).

\subsection{Viscosity measurement}

A Schott-Ubbelohde Size IV Capillary Viscometer was used to measure the viscosity of different PVA concentrations. Briefly: the viscometer was kept at $25^{\circ} \mathrm{C}$ by a water bath covering the pre-run sphere to ensure temperature homogeneity across the glass tubing. The liquid reservoir was filled with PVA solutions from 1/100 to 10/100 PVA/water mass ratio. Values were converted into w/w percentages for ease of comparison of the results. Sample preparation was performed as described in the manufacturer's instructions provided with the device, with falling time defined as the time needed for the sample to run through two reference marks provided in the viscometer. Falling time was then converted to viscosity, expressed in $\mathrm{CP}$, by conversion $\eta=t \times K$, where $K$ is an experimentally determined constant provided by the manufacturer whose value was $9.917 \mathrm{cP}$.

\section{Results and Discussion}

\subsection{PLLA/MNP microparticle characterization}

The morphology of PLLA microparticles with embedded MNP was observed by FESEM, as seen in Figure 1. The particle diameter is observed to be between 5 and $50 \mu \mathrm{m}$. Interestingly, the PLLA microspheres without encapsulated MNP show a very smooth surface (inset in Figure 1), whereas the surface of PLLA/MNP microspheres appears rough. To determine if this change in surface topography could be ascribed to the accumulation of MNPs at the surface, the cross-section of the microspheres was observed by using a Focused Ion Beam, FIB, equipment that cuts the micrparticles inside the FESEM. Figure 2a shows one of the PLLA/MNPs after FIB cut, while Figure $2 b$ shows a cross-section view. MNPs with diameters in the range of 10 to $30 \mathrm{~nm}$ appear well dispersed through a PLLA matrix. In the synthesis of PLLA/MNP microspheres, the first stage was the suspension of MNPs in PLLA solution in chloroform under continuous stirring. Interestingly enough this dispersion 
is maintained during formation of microparticles in the water-oil emulsion. Thus, it seems that the surface roughness is not a consequence of nanoparticle agglomeration. It should be ascribed to a change in PLLA crystallinity as will be shown below using DSC.

[Figure 1]

[Figure 2]

Differential scanning calorimetry scans were conducted before and after argon plasma treatment (Figure 3). The thermogram of PLLA microspheres without MNPs corresponds to an amorphous polymer. The glass transition appears around $60^{\circ} \mathrm{C}$ accompanied by a large overshoot due to physical ageing. After the glass transition, when the PLLA chains acquire enough mobility, crystallization takes place in two steps shown in the thermogram by two exothermal peaks, one of them between $70^{\circ} \mathrm{C}$ and $120^{\circ} \mathrm{C}$ and the other one in the range between $150^{\circ} \mathrm{C}$ and $165^{\circ} \mathrm{C}$, followed by melting. This behavior in amorphous PLLA has been described previously by others. ${ }^{16}$ The total area calculated in the thermogram using a straight baseline between $70^{\circ} \mathrm{C}$ and $195^{\circ} \mathrm{C}$ is nearly zero, showing that the sample at the start of the scan was amorphous. The scan recorded in PLLA/MNPs is quite different, the exothermals are not shown, so the melting peak is due to crystals already present in the sample at the beginning of the scan. Crystallinity values are listed in Table 1, showing that argon plasma treatments had no significant influence on crystallinity. The increased crystallinity of the MNP-loaded samples might be attributed to a nucleation effect: the nanoparticles, which were crystalline in nature, could act as nuclei for the formation of further crystals, thus speeding up the limiting phase of the crystallization process, which occurred during solvent evaporation in the emulsion.

\section{[Figure 3]}

A second heating scan was recorded after cooling the sample from the melt. In these measurements PLLA crystallization could take place from the melt during cooling. It is well known that PLLA crystallization is quite slow and cooling, even at moderate cooling rates, yields an amorphous polymer. ${ }^{12,39,30}$ It is worth noting that the nucleation effect of MNPs is not shown in the second heating scans (the second scans of the PLLA and PLLA/MNPs are similar to each other). This means that 
MNPs act as nucleation points during crystallization from solution but not during crystallization from the melt.

\section{[Table 1]}

The PLLA glass transition temperature ( $\left.T_{g}\right)$ and heat capacity jump at $T_{g}, \Delta c_{p}$, show no variation with plasma treatment. However, in the first heating scan, particles without MNP displayed both higher $\Delta c_{p}$ and $T_{g}$; this is accrued to a higher amount of amorphous phase. Nevertheless, in the second heating scan the glass transition occurs at the same temperature and with similar peak profiles. This behavior is concordant with the initial crystallinity: The MNP-loaded samples had lower amounts of glass phase, which is responsible for the glass transition.

A Gel Permeation Chromatography assay was carried out for the PLLA microparticles with and without previous argon gas treatment. The inset in the Figure 3 shows the molecular weight distribution of one sample, representative of both treated and non-treated argon plasma. The molecular weight of all samples is in the range of $230-260 \mathrm{kDa}$, which broadly corresponds to manufacturer's data. The chromatograms of samples subjected or not to plasma treatment completely superpose. No sign of chain cleavage due to plasma interaction was found.

\subsection{Assessment of PLLA/MNP motion in viscous media}

Figure 4 shows that the viscosity of the PVA solutions increases exponentially with the polymer concentration. Intrinsic viscosity was extrapolated at point 0 , resulting in a value of $3.57 \mathrm{dL} / \mathrm{g}$.

\section{[Figure 4]}

Testing of the deformation induced in the neighborhood of the particle due to magnetic field was performed on different concentrations of PVA solution containing PLLA/MNPs. To represent the different phases of ECM production, water and different concentrations of the PVA solution were tested. Samples were placed between two electromagnets where the applied magnetic flux density was in the range of 20-50 mT, depending on the current intensity (see Figure 5). 


\section{[Figure 5]}

The magnetic force acting on a magnetic dipole $\vec{m}$ can be written as:

$$
\overrightarrow{F_{M}}=(\vec{m} \cdot \vec{v}) \cdot \vec{B}
$$

where $\vec{B}$ is the magnetic flux density and $\vec{\nabla}=\left(\frac{\partial}{\partial x}, \frac{\partial}{\partial y}, \frac{\partial}{\partial z}\right)$ the nabla operator. As the microparticles were immersed in a fluid, the hydrodynamic drag force, $F_{D}$, exerted on spherical objects with very small Reynolds number (very small particles) in a viscous fluid, obeys the Stokes' law:

$$
F_{D}=6 \pi \eta R v
$$

where $\eta$ is the fluid viscosity, $R$ the radius of the spherical particle in motion, and $v$ its velocity. Solving the equation of forces, a limit velocity is obtained (when both forces are equal).

This velocity limit has been experimentally measured, for different configurations, placing the samples between the north south electromagnet poles on a microscope stage. Imagery was recorded and analyzed by algorithms based on image threshold techniques. ${ }^{25} \mathrm{~A}$ high contrast was found between the PLLA/MNP microparticles and the fluid resulting in a low computation power. Tracking was done on the centroid of the area, bordering the perimeter of the microparticle. This technique is robust, providing the centroid position with subpixel accuracy. The recording started with no magnetic field applied, to be able to detect the fluid motion and, once the magnetic field was applied, to determine the particle velocity with respect to the surrounding fluid. Results of the terminal velocity are shown in Table 2. In all cases terminal velocity is reached very quickly, as could be deduced from the linearity of the position respect to the time. A linear dependence of the terminal velocity on the viscosity was observed. When the PLLA/MNPs where placed in the most viscous fluid, PVA-4\%, the motion was very slow and higher intensity (hence higher magnetic force) was required.

An estimation of the applied force acting on the microparticles was obtained from the results of the terminal velocity by applying Eq. 6 . Results are shown in Table 2 .

[Table 2] 
An oscillating motion of the PLLA/MNPs was produced by applying an alternating current to the electromagnets. This generated a gradient of the magnetic field in the gap that changed direction periodically and, therefore, according to equation 5 an oscillating force was acting on the particles producing an oscillating motion. Again, the oscillating motion was recorded and, following the same procedure, the maximum displacement was obtained (twice the amplitude of the oscillating motion). The dependence of the amplitude on viscosity is what one could expect from the theory when both PVA hydrogels were compared. PLLA/MNPs in water form aggregates and no single particle could be selected; the diameter indicated in Table 2 is the approximate size. One of the recorded videos, which corresponds to PVA $2 \%$, can be seen in the following link: http://politube.upv.es/play.php?vid=66942.

\section{VIDEO}

\subsection{Scaffold /hydrogel construct}

In Figure 6a the PLLA microparticles embedded in the PVA hydrogel after 6 cycles of freezing and thawing, and both inside the pores of the PLLA scaffold can be seen. Microparticles are well dispersed in the gel. Figure $6 \mathrm{~b}$ corresponds to the same sample observed at higher magnification. As seen in the picture, the gel completely fills the scaffold pores and the microparticles effectively entered the scaffold and are distributed in the PVA hydrogel. Effectiveness of the PVA filling of the pores of the PCL scaffold was quantified based on mass, density and volume of the samples, as explained in the experimental section (Equations 1, 2 and 3).

Porosity calculations give a value close to 0 , confirming complete filling of the pores of the scaffold, in accordance with SEM observation.

\section{[Figure 6]}

Unconfined compression tests of the PCL scaffolds filled with PVA hydrogel with and without PLLA microspheres, and after 6 cycles of freezing and thawing, were performed to evaluate the mechanical influence of the microspheres. Figure $7 \mathrm{a}$ shows the stress-strain representation from the compression test of the $15 \%$ and $20 \%$ PCL scaffolds with PVA hydrogel with and without microparticles. Figure $7 \mathrm{~b}$ 
shows the elastic modulus calculated from the linear zone of the stress-strain representation for the different samples. The elastic modulus increases with increasing $\mathrm{PCL}$ content in the PCL/dioxane solution prepared for scaffold manufacturing, which is in accordance with previous studies. ${ }^{37}$ When introducing the PLLA microspheres in the PVA hydrogel, it can be seen that the modulus does not increase, compared to the samples without polymer microspheres. This indicates that the microparticles do not impede water passage through the PVA hydrogel which could be a cause of mechanical modulus increase, as observed in other studies by the group. ${ }^{35,37}$

\section{[Figure 7]}

\section{Conclusions}

PLLA microspheres containing magnetic nanoparticles with diameters between 10 and $30 \mathrm{~nm}$ have been produced. The emulsion process starting with a good dispersion of nanoparticles in the PLLA solution in chloroform maintained homogeneity of MNP distribution without clustering. MNPs acted as a nucleating agent for PLLA during crystallization from the chloroform solution during microspheres formation but the same effect was not observed when PLLA containing MNPs crystallized from the melt. PLLA crystallization made the surface of the microspheres to become rough due to the presence of PLLA spherulites. The application of a magnetic field to PLLA/MNP microspheres produced their displacement in a more or less viscous solution, thus inducing a deformation field in the dispersion media. The size of the PLLA/MNP microspheres enabled introducing them into the pores of a macroporous scaffold aimed at tissue engineering. This study shows that the microspheres could eventually be introduced into scaffolds together with a cell suspension in order to mechanically stimulate the cells during regeneration.

\section{Acknowledgements}

The authors gratefully acknowledge the financial support from the Spanish Ministry of Economy and Competitiveness through the MAT2013-46467-C4-1-R project, including the Feder funds. CIBER-BBN is an initiative funded by the VI National 
R\&D\&I Plan 2008-2011, Iniciativa Ingenio 2010, Consolider Program. CIBER Actions are financed by the Instituto de Salud Carlos III with assistance from the European Regional Development Fund. The authors thank "Servicio de Microscopia Electrónica" of Universitat Politècnica de València for their invaluable help. The translation of this paper was funded by the Universitat Politècnica de València, Spain.

\section{References:}

1. Appelman, T., J. Mizrahi, J. Elisseeff and D. Seliktar. The influence of biological motifs and dynamic mechanical stimulation in hydrogel scaffold systems on the phenotype of chondrocytes. Biomaterials 32:1508-1516, 2011.

2. Banes, A. J., M. Tsuzaki, J. Yamamoto, T. Fischer, B. Brigman, T. Brown and L. Miller. Mechanoreception at the cellular level: the detection, interpretation, and diversity of responses to mechanical signals. Biochem. Cell Biol. 73:349-365, 1995.

3. Bausch, A. R., U. Hellerer, M. Essler, M. Aepfelbacher and E. Sackmann. Rapid stiffening of integrin receptor-actin linkages in endothelial cells stimulated with thrombin: a magnetic bead microrheology study. Biophys. J. 80:2649-2657, 2001.

4. Brady, M. A., S. D. Waldman and C. R. Ethier. The application of multiple biophysical cues to engineer functional neocartilage for treatment of osteoarthritis. Part I: cellular response. Tissue Eng. Part B Rev. 21:1-19, 2015.

5. Crick, F. H. C. and A. F. W. Hughes. The physical properties of cytoplasm: a study by means of the magnetic particle method. Exp. Cell Res. 1:37-80, 1950. 
6. Eckstein, F., B. Lemberger, C. Gratzke, M. Hudelmaier, C. Glaser, K. H. Englmeir and M. Reiser. In vivo cartilage deformation after different types of activity and its dependence on physical training status. Ann. Rheum. Dis. 64:291-295, 2005.

7. Eyre, D. Collagen of articular cartilage. Arthritis Res.4:30-35, 2002.

8. Garlotta, D. A literature review of poly(lactic acid). J. Polym. Env. 9:63-84, 2001.

9. Gillard, G. C., H. C. Reilly, P. G Bell-Booth and M. H. Flint. The influence of mechanical forces on the glycosaminoglycan content of the rabbit flexor digitorum profundus tendon. Connect. Tissue Res. 7:37-46, 1979.

10. Grant, C., P. Twigg, A. Egan, A. Moody, D. Eagland, N. Crowther and S. Britland. Poly(vinyl alcohol) hydrogel as a biocompatible viscoelastic mimetic for articular cartilage. Biotechnol. Prog.22:1400-1406, 2006.

11. Hassan, C. M. and N. A. Peppas. Structure and applications of poly(vinyl alcohol) hydrogels produced by conventional crosslinking or by freezing/thawing methods. Adv. Polym. Sci.153:37-65, 2000.

12. Hernández, F., J. Molina Mateo, F. Romero Colomer, M. Salmerón Sánchez, J. L. Gómez Ribelles and J. Mano. Influence of low-temperature nucleation on the crystallization process of poly(L-lactide). Biomacromolecules 6:3291-3299, 2005.

13. Hutmacher, D. W. Scaffolds in tissue engineering bone and cartilage. Biomaterials 21:2529-2543, 2000.

14. Ingber, D. E. Tensegrity: the architectural basis of cellular mechanotransduction. Annu. Rev. Physiol. 59:575-599, 1997. 
15. Khan, S. and M. P. Sheetz. Force effects on biochemical kinetics. Annu. Rev. Biochem. 66:785-805, 1997.

16. Kovacs, A., J. Aklonis, J. Hutchinson and A. Ramos. Isobaric volume and enthalpy recovery of glasses. II. A transparent multiparameter theory. J. Polym. Sci., Polym. Phys. 17:1097-1162, 1979.

17. Labet, M., and W. Thielemans. Synthesis of polycaprolactone: a review. Chem. Soc. Rev. 38:3484-3504, 2009.

18. Langer, R. and J. P. Vacanti. Tissue engineering. Science 260:920-926, 1993.

19. Lebourg, M., J. Suay Antón and J. L. Gómez Ribelles. Porous membranes of PLLA-PCL blend for tissue engineering applications. Eur. Polym. J. 44:2207-2218, 2008.

20. Li, F., Y. L. Su, D. F. Shi and C.T. Wang. Comparison of human articular cartilage and polyvinyl alcohol hydrogel as artificial cartilage in microstructure analysis and unconfined compression. Adv. Mater. Res. Trans. Tech. Publ. 87:188-193, 2010.

21. Li, L., G. Yang, J. Li, S. Ding and S. Zhou. Cell behaviors on magnetic electrospun poly- D , L -lactide nano fi bers. Mater. Sci. Eng. C 34:252-261, 2014.

22. Mano, J. F., J. L. Gómez Ribelles, N. M. Alves and M. Salmerón Sanchez. Glass transition dynamics and structural relaxation of PLLA studied by DSC: Influence of crystallinity. Polymer 46:8258-8265, 2005.

23. Mow, V. C., A. Ratcliffe and A. R. Poole. Cartilage and diarthrodial joints as paradigms for hierarchical materials and structures. Biomaterials 13:67-97, 1992. 
24. Mow, V. C. and R. Huiskes. Basic Orthopaedic Biomechanics and Mechanobiology. Philadelphia: Lippincott Williams and Wilkins, 2005.

25. Nobuyuki, O. A threshold selection method from gray-level histograms. Automatica 11:23-27, 1975.

26. Panadero, J. A., L. Vikingsson, J. L. Gomez Ribelles, S. Lanceros-Mendez and V. Sencadas. In vitro mechanical fatigue behaviour of poly- $\varepsilon$-caprolactone macroporous scaffolds for cartilage tissue engineering. Influence of pore filling by a poly(vinyl alcohol) gel. J. Biomed. Mater. Res. Part B - Appl. Biomater. 103:10371043, 2015.

27. Pommerenke, H., E. Schreiber, F. Durr, B. Nebe, C. Hahnel, W. Moller and J. Rychly. Stimulation of integrin receptors using a magnetic drag force device induces an intracellular free calcium response. Eur. J. Cell Biol. 70:157-164, 1996.

28. Quinn, T. M., A. J. Grodzinsky, M. D. Buschmann, Y. J. Kim and E. B. Hunziker. Mechanical compression alters proteoglycan deposition and matrix deformation around individual cells in cartilage explants. J. Cell Sci. 111:573-583, 1998.

29. Roughley, P. J. and E. R Lee. Cartilage proteoglycans: structure and potential functions. Microsc Res Tech. 28:385-397, 1994.

30. M. Salmerón Sánchez, B. M. Vincent, G. Vanden Poel and J. L. Gómez-Ribelles. Effect of the cooling rate on the nucleation kinetics of poly(L-lactic acid) and its influence on morphology. Macromolecules 40:7989-7997, 2007.

31. Santamaría, V. A., H. Deplaine, D. Mariggió, A. R. Villanueva-Molines, J. M. García-Aznar, J. L. Gómez Ribelles, M. Doblaré, G. Gallego Ferrer and I. Ochoa. 
Influence of the macro and micro-porous structure on the mechanical behavior of poly (L-lactic acid) scaffolds. J. Non Cryst. Solids. 358:3141-3149, 2012.

32. Valberg, P. A. and D. F. Albertini. Cytoplasmic motions, rheology, and structure probed by a novel magnetic particle method. J. Cell Biol. 101:130-140, 1985.

33. Valberg, P. A. and H. A. Feldman. Magnetic particle motions within living cells. Measurement of cytoplasmic viscosity and motile activity. Biophys. J. 52:551-561, 1987.

34. Valhmu, W. B., E. J. Stazzone, N. M. Bachrach, F. Saed-Nejad, S. G. Fischer, V. C. Mow and A. Ratcliffe. Load-controlled compression of articular cartilage induces a transient stimulation of aggrecan gene expression. Arch. Biochem. Biophys. 353:2936, 1998.

35. Vikingsson, L., G. Gallego Ferrer, J. A. Gómez-Tejedor and J. L. Gómez Ribelles. An in vitro experimental model to predict the mechanical behaviour of macroporous scaffolds implanted in articular cartilage. J. Mech. Behav. Biomed. Mater. 32:125131, 2014.

36. Vikingsson, L., J. A. Gomez-Tejedor, G. Gallego Ferrer and J. L. Gomez Ribelles. An experimental fatigue study of a porous scaffold for the regeneration of articular cartilage. J. Biomech. 48:1310-1317, 2015.

37. Vikingsson, L., B. Claessens, J. A. Gómez-Tejedor, G. Gallego Ferrer and J. L. Gómez Ribelles. Relationship between micro-porosity, water permeability and mechanical behavior in scaffolds for cartilage engineering. J. Mech. Behav. Biomed. Mater. 48:60-69, 2015. 
38. Wang, N. and D. E. Ingber. Probing transmembrane mechanical coupling and cytomechanics using magnetic twisting cytometry. Biochem. Cell Biol. 73:327-335, 1995.

39. Wang, Y., J. L. Gómez Ribelles, M. Salmerón Sánchez and J. F. Mano. Morphological contribution to glass transition in poly(L-lactic acid). Macromolecules 38:4712-4718, 2005. 


\section{Tables}

\section{Table 1}

Table 1. The $T_{g}$ and crystallinity (\%) for PLLA microspheres, according to type of plasma treatment and microparticles load. All values for the second scans equaled zero or negative crystallinity values.

\begin{tabular}{|c|c|c|c|c|c|c|}
\hline Load & $\begin{array}{l}\text { Plasma } \\
\text { cycles }\end{array}$ & $\begin{array}{c}\mathrm{T}_{\mathrm{g}}\left({ }^{\circ} \mathrm{C}\right) \\
\text { fist scan }\end{array}$ & $\begin{array}{l}T_{g}\left({ }^{\circ} \mathrm{C}\right) \\
\text { second } \\
\text { scan }\end{array}$ & $\begin{array}{c}\Delta c_{p}\left(\mathrm{~J} /\left(g^{\circ} \cdot{ }^{\circ} \mathrm{C}\right)\right. \\
\text { at } \mathrm{T}_{\mathrm{g}} \\
\text { (first scan) }\end{array}$ & $\begin{array}{l}\Delta \mathrm{c}_{\mathrm{p}}(\mathrm{J} /(\mathrm{g} \cdot \\
\left.{ }^{\circ} \mathrm{C}\right) \text { at } \mathrm{T}_{\mathrm{g}} \\
\text { (second } \\
\text { scan) }\end{array}$ & $\begin{array}{l}\text { Initial crystallinity } \\
\text { (\%) } \\
\text { (first scan) }\end{array}$ \\
\hline \multirow{3}{*}{$\begin{array}{l}5 \% \\
\text { MNP }\end{array}$} & 0 & $57.4 \pm 0.5$ & \multirow{3}{*}{$55.1 \pm 1.8$} & $0.14 \pm 0.05$ & \multirow{3}{*}{$0.41 \pm 0.02$} & $33.8 \pm 4.2$ \\
\hline & 1 & $56.4 \pm 0.1$ & & $0.16 \pm 0.02$ & & $35.7 \pm 3.3$ \\
\hline & 2 & $58.1 \pm 1.3$ & & $0.15 \pm 0.06$ & & $34.2 \pm 7.0$ \\
\hline \multirow{3}{*}{$\begin{array}{c}\text { No } \\
\text { MNP }\end{array}$} & 0 & $60.1 \pm 1.3$ & \multirow{3}{*}{$55.1 \pm 0.5$} & $0.54 \pm 0.03$ & \multirow{3}{*}{$0.44 \pm 0.03$} & - \\
\hline & 1 & $60.9 \pm 0.4$ & & $0.53 \pm 0.09$ & & - \\
\hline & 2 & $60.1 \pm 0.4$ & & $0.48 \pm 0.00$ & & - \\
\hline
\end{tabular}




\section{Table 2}

Table 2: Experimental parameters and results of terminal velocity and maximum displacement when magnetic forces are applied on PLLA/MNPs immersed in a fluid. *Particles tend to form agglomerates when they are dispersed in a low viscosity fluid, even with plasma treatment.

\section{Terminal velocity}

\begin{tabular}{|c|c|c|c|c|c|c|}
\hline Fluid & $\begin{array}{c}\text { Viscosity } \\
(\mathbf{P a} \cdot \mathbf{s})\end{array}$ & $\begin{array}{c}\text { Electro } \\
\text { magnet } \\
\text { configuration }\end{array}$ & $\begin{array}{c}\text { Current } \\
(\mathbf{A})\end{array}$ & $\begin{array}{c}\text { Particle } \\
\mathbf{s i z e} \\
(\boldsymbol{\mu m})\end{array}$ & $\begin{array}{c}\text { Terminal } \\
\text { velocity } \\
(\boldsymbol{\mu m} / \mathbf{s})\end{array}$ & $\begin{array}{c}\text { Force } \\
(\mathbf{p N})\end{array}$ \\
\hline Water & $10^{-3}$ & $\mathrm{~N}-\mathrm{S}$ & 1 & $38 \pm 3$ & $188 \pm 1$ & $67 \pm 6$ \\
\hline PVA 2\% & $14 \cdot 10^{-3}$ & $\mathrm{~N}-\mathrm{S}$ & 1 & $32 \pm 3$ & $6.4 \pm 0.02$ & $27 \pm 3$ \\
\hline PVA 4\% & $56 \cdot 10^{-3}$ & $\mathrm{~N}-\mathrm{S}$ & 2.8 & $15 \pm 3$ & $0.38 \pm 0.002$ & $3.1 \pm 0.6$ \\
\hline
\end{tabular}

\section{Oscillating motion}

\begin{tabular}{|c|c|c|c|c|c|c|}
\hline Fluid & $\begin{array}{c}\text { Viscosity } \\
(\mathbf{P a} \cdot \mathbf{s})\end{array}$ & $\begin{array}{c}\text { Electro } \\
\text { magnet } \\
\text { configuration }\end{array}$ & $\begin{array}{c}\text { Current } \\
(\mathbf{A}) \\
\text { Frequency } \\
(\mathbf{H z})\end{array}$ & $\begin{array}{c}\text { Particle size } \\
(\boldsymbol{\mu m})\end{array}$ & $\begin{array}{c}\text { Maximum } \\
\text { displacement }\end{array}$ & \\
\hline Water & $10^{-3}$ & $\mathrm{~N}-\mathrm{N}$ & $\begin{array}{c}4 \mathrm{~A} \\
1 \mathrm{~Hz}\end{array}$ & $\begin{array}{c}\text { particles } \\
\text { agglomerates }\end{array}$ & $455 \pm 5$ & \\
\hline PVA 2\% & $14 \cdot 10^{-3}$ & $\mathrm{~N}-\mathrm{N}$ & $\begin{array}{c}4 \mathrm{~A} \\
1 \mathrm{~Hz}\end{array}$ & $15 \pm 3$ & $0.32 \pm 0.05$ & \\
\hline PVA_4\% & $56 \cdot 10^{-3}$ & $\mathrm{~N}-\mathrm{N}$ & $\begin{array}{c}4 \mathrm{~A} \\
1 \mathrm{~Hz}\end{array}$ & $14 \pm 3$ & $0.08 \pm 0.01$ & \\
\hline
\end{tabular}




\section{Figures}

\section{Figure 1}

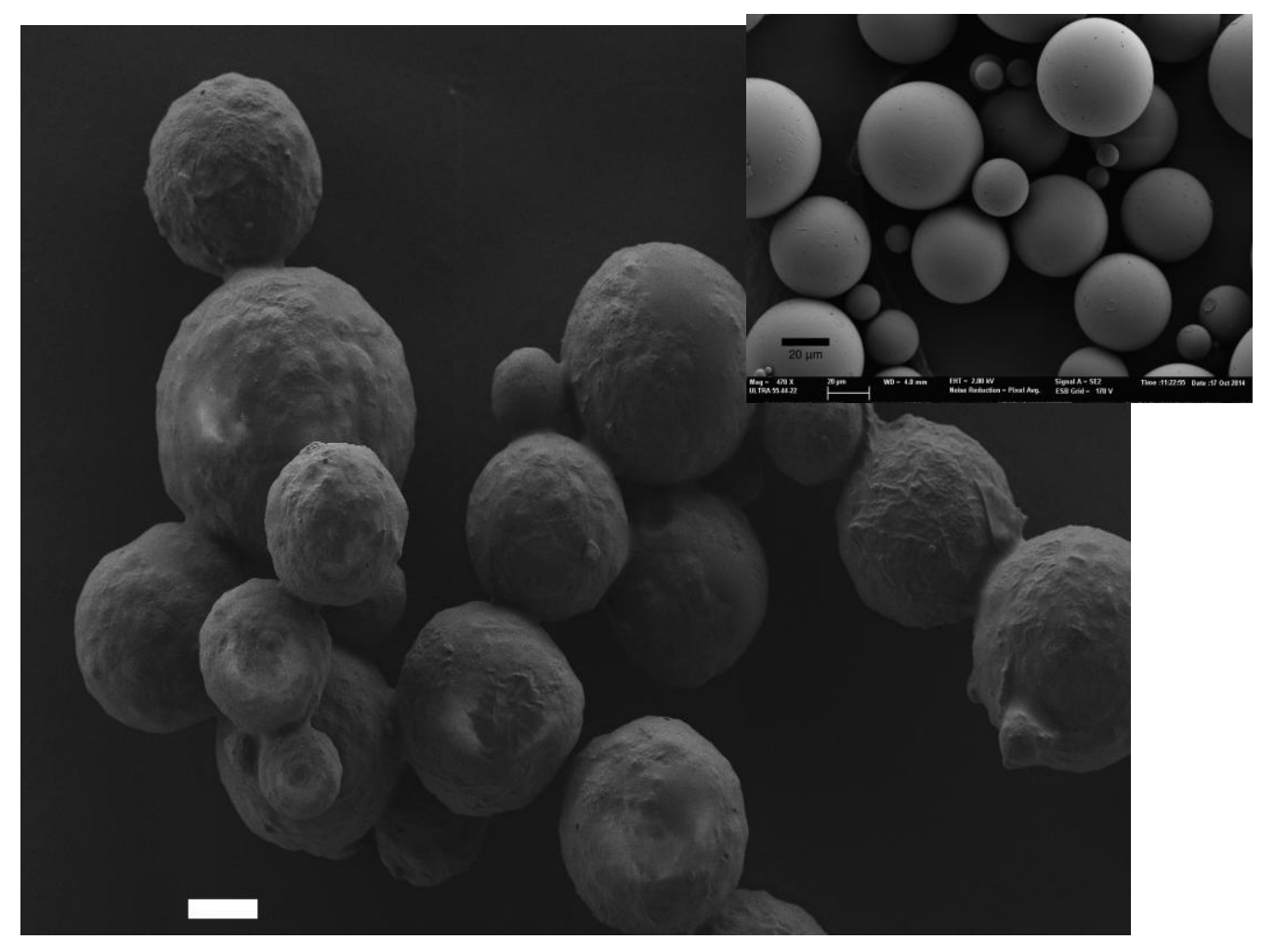

Figure 1. The morphology of PLLA microparticles filled with MNP (PLLA/MNP) observed in FESEM. Scale bar $10 \mu \mathrm{m}$. The inset shows PLLA microparticles without MNP. Dimension bar in the inset picture is $20 \mu \mathrm{m}$. The particles were observed after plasma treatment. 


\section{Figure 2}

a)

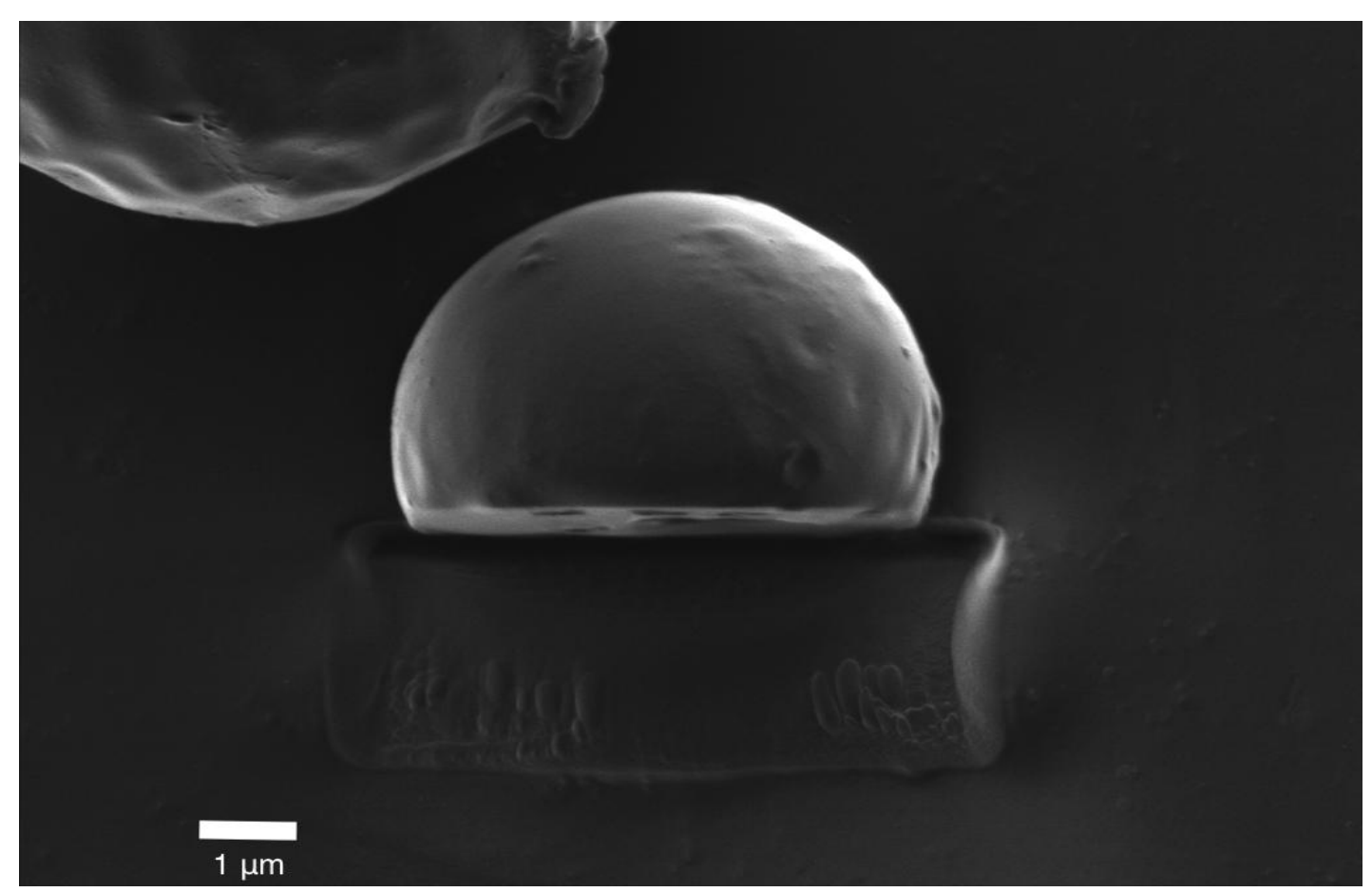

b)

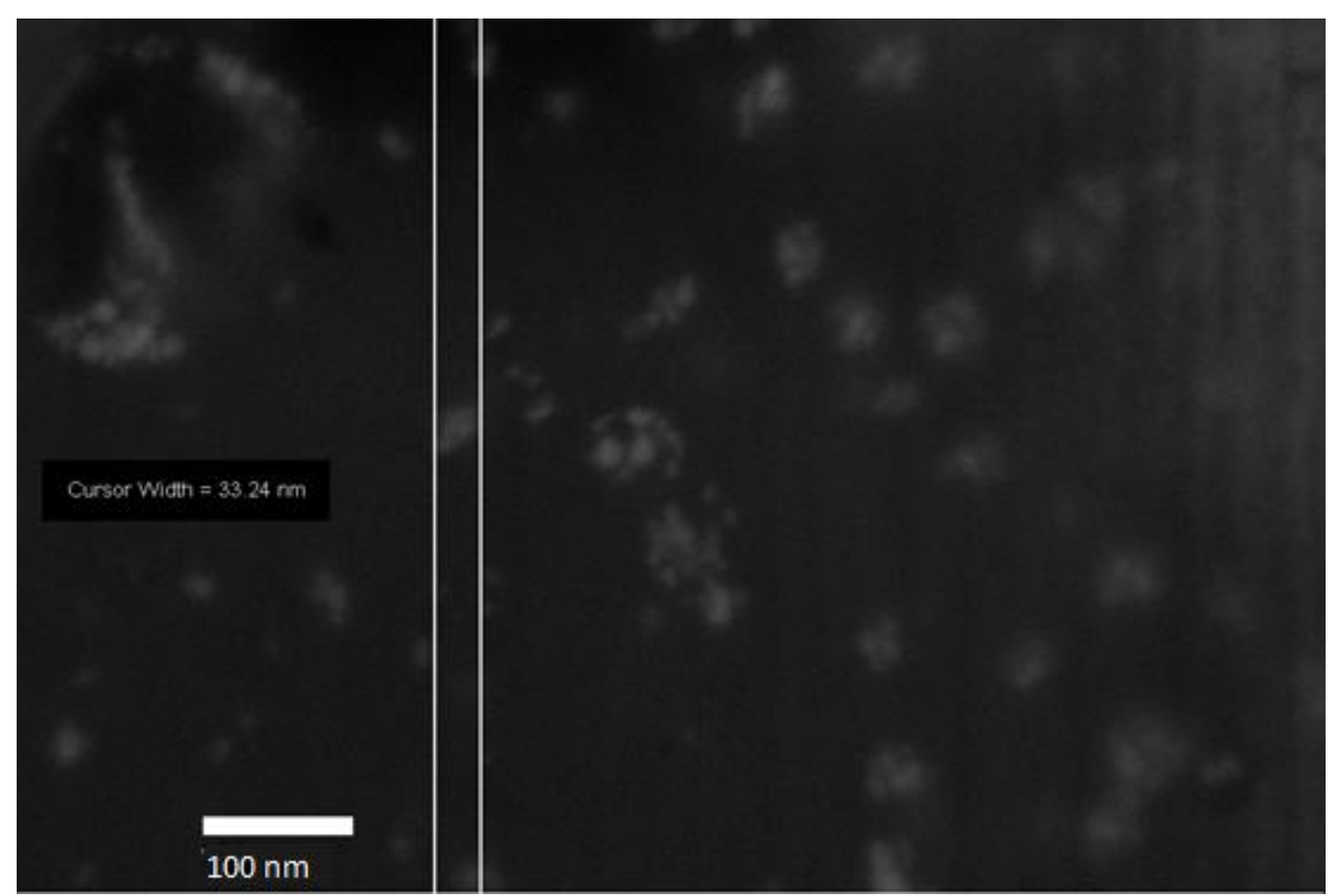

Figure 2. (a) A PLLA/MNP microparticle observed in the FESEM microscope after FIB cutting to observe the internal structure. A very small particle in comparison to 
the mean was chosen for easy cutting. Scale bar $1 \mu \mathrm{m}$. (b) The MNPs are seen inside the matrix of the PLLA sphere. Scale bar $100 \mathrm{~nm}$. The particle was observed without plasma treatment.

\section{Figure 3}

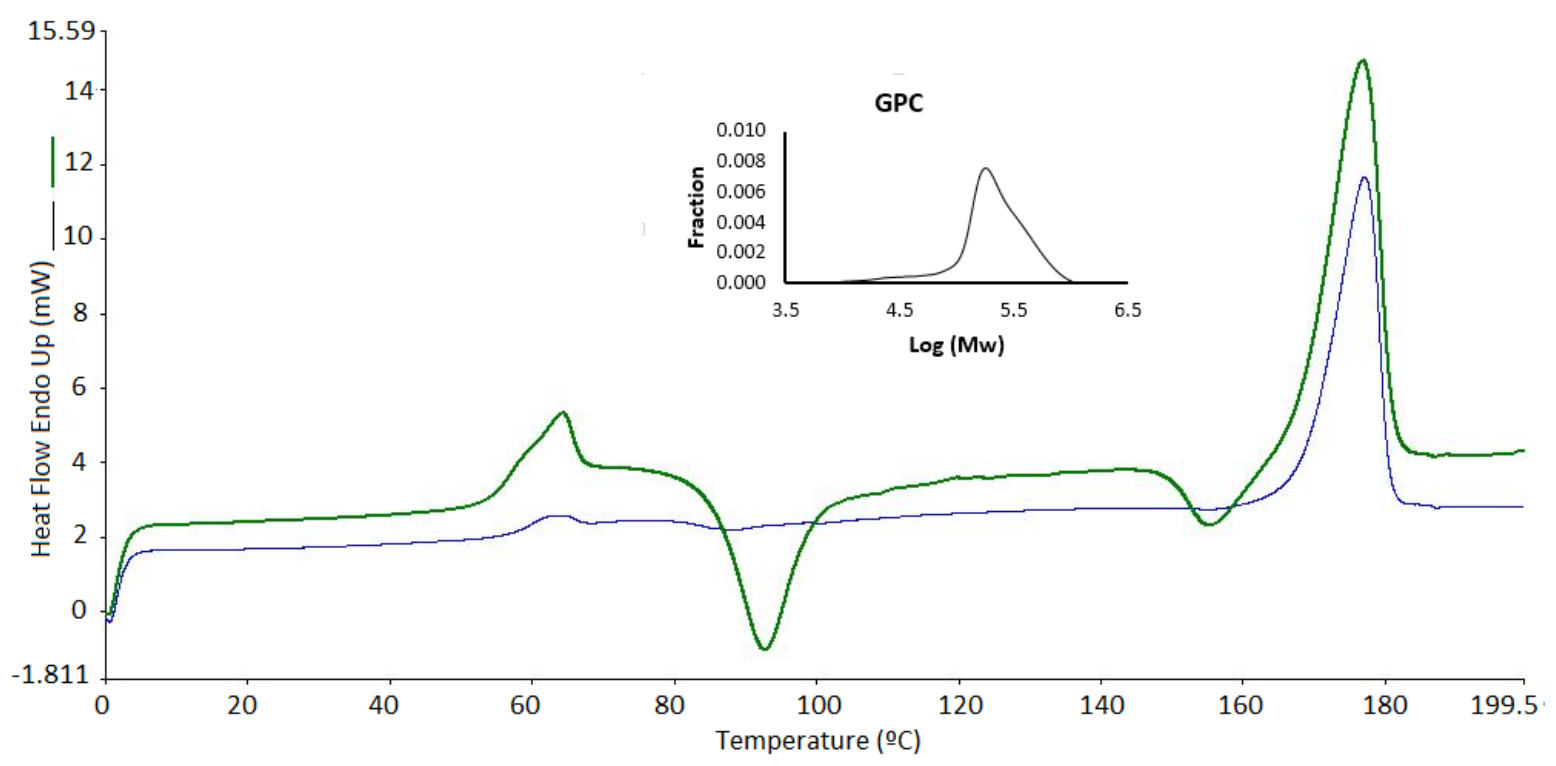

Figure 3. First DSC heating scan for the PLLA microspheres with 5\% MNP (blue line) and without MNP (green line), both samples without plasma treatment. The inset shows the GPC results of the PLLA microspheres. A single curve was portrayed as there was a total overlap between the different curves with and without plasma treatment. 


\section{Figure 4}

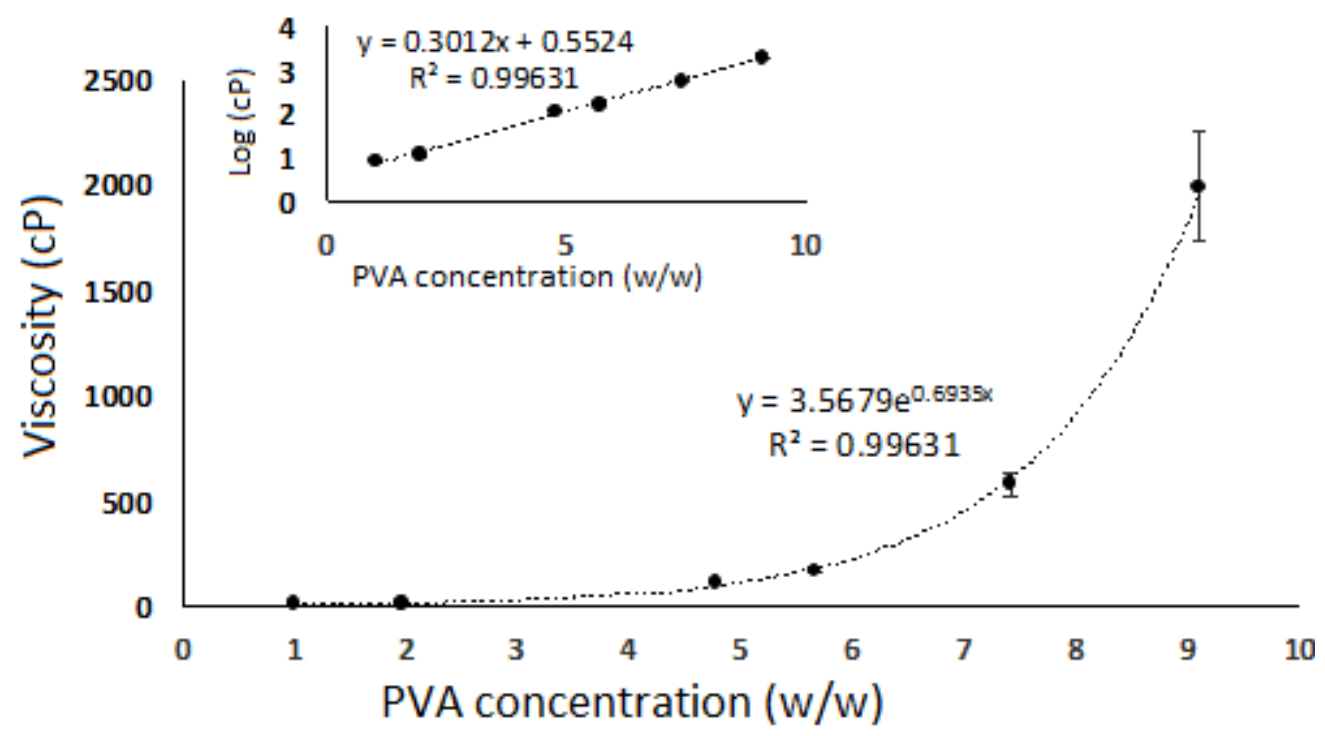

Figure 4. Viscosity of PVA solutions according to the viscosity (cP) measured by falling time, plotted against PVA concentration. The figure also shows the logarithmic relation between viscosity and polymer concentration.

\section{Figure 5}

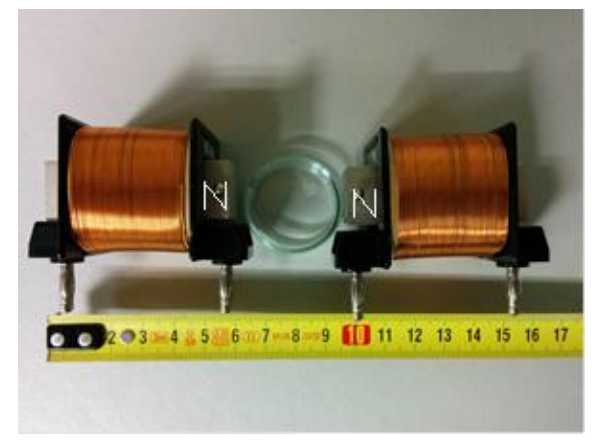

Figure 5. Experimental setup. Water or PVA solution was placed in a Petri dish between two electromagnets. The magnetic flux density in the gap depends on the current intensity. 


\section{Figure 6}

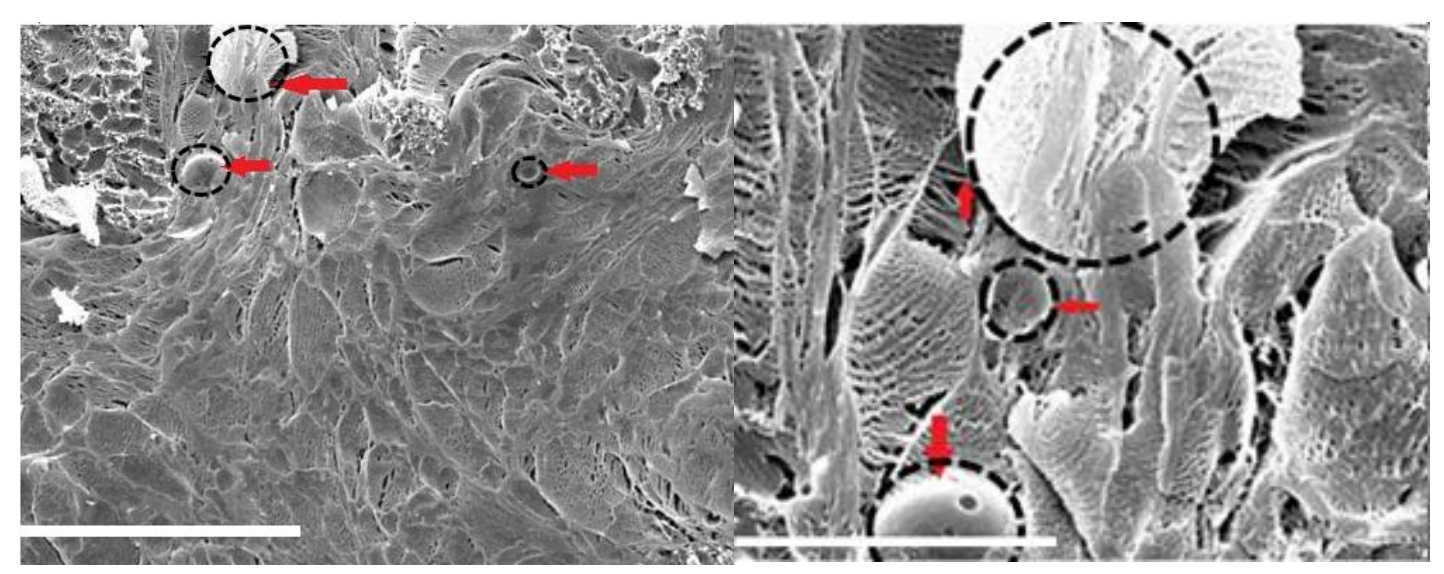

Figure 6. (a) SEM pictures of the PLLA microparticles embedded in the PCL scaffold filled with PVA hydrogel after 6 cycles of freezing and thawing. Scale bar $100 \mu \mathrm{m}$. (b) Magnification of the previous image 6a). Scale bar $30 \mu \mathrm{m}$. 


\section{Figure 7}

a)

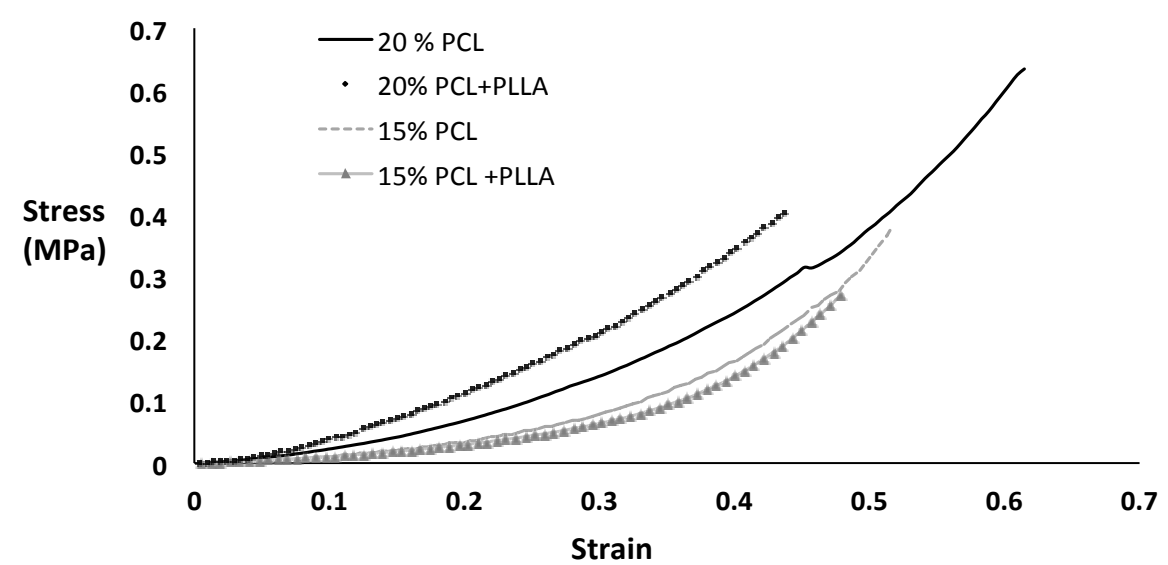

b)

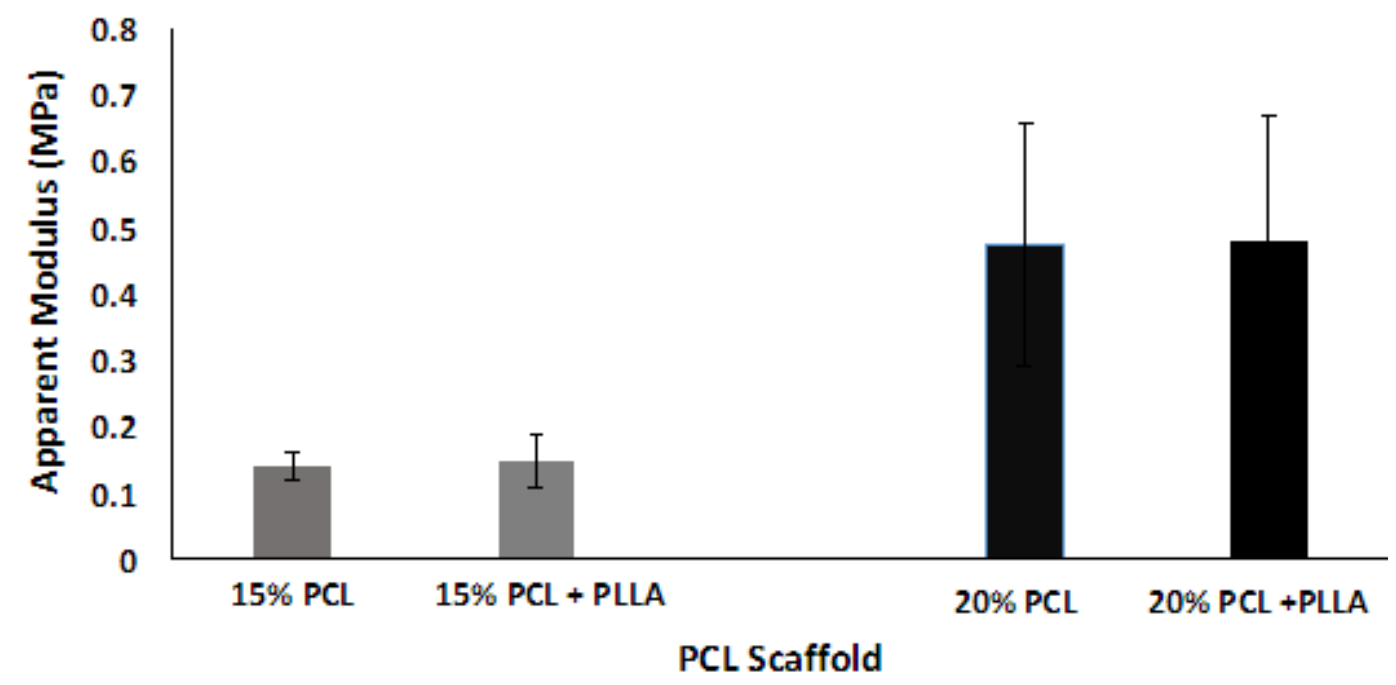

Figure 7. (a) The stress-strain graph from the unconfined compression tests of the 15 and 20\% PCL scaffolds filled with PVA hydrogel with and without PLLA microparticles. (b) The apparent elastic modulus calculated in the linear zone of the stress-strain curve for the scaffolds. 